\title{
Handbook of Model Predictive Control
}

\author{
by Saša V. Raković and William S. Levine (Editors)
}

\author{
Reviewed by GABRIELE PANNOCCHIA
}

\begin{abstract}
Model Predictive Control (MPC), also referred to as Receding Horizon Control, is a wide class of optimizationbased control methods and algorithms based on a very simple and powerful idea. A dynamical model of the system to be controlled is used to make a finite-horizon prediction of the state evolution as a function of the input sequence, the optimal input sequence is computed by maximizing some performance measure while respecting state/input constraints, only the first action of the optimal control sequence is actually implemented, and at the next decision time the whole procedure is repeated taking into account the new current state of the system. MPC algorithms were originated in the ' 70 s to face the increasing economic optimization needs and challenges of the refinery industries [1], [2]. After an initial reluctance of the academia, at that time mainly focused on multivariable techniques $(\mathrm{H}-$ infinity, LQG , ...) and adaptive control, from the early ' 90 s it witnessed a never seen explosion of research contributions [3], [4] and industrial applications [5].
\end{abstract}

The initial MPC algorithms were implemented using discrete-time linear convolution models identified from data, but later research studies and computational software specifically developed for MPC made use of state-space models (linear or nonlinear, continuous-time or discrete-time). These developments broadened the scope of MPC to a plethora of application fields where MPC was for long time considered inapplicable due to its computational demands. Meanwhile, theory of linear and nonlinear MPC grew steadily, thus reaching a high level of maturity [6], [7], also making MPC ubiquitous in advanced control courses. Furthermore, significant advances in stochastic modeling, numerical optimization, dynamical system analysis, and hardware implementations guarantee always new opportunities for MPC based approaches. It is therefore important to have an up-to-date and wide reference, as the Handbook of Model Predictive Control, edited by Raković and Levine.

The Handbook of Model Predictive Control includes twentyseven chapters, coherently organized into three parts. Part I is on Theory and comprises twelve chapters, ranging from basic MPC theory to advanced studies and MPC formulations. Part II, on Computations, includes eight chapters and covers numerical implementation of MPC related optimization algorithms. Part III presents Applications in numerous fields where MPC is becoming successful, such as automotive, power and energy systems, healthcare and finance.

\section{CONTENTS}

\section{Part I: Theory}

The first chapter introduces the basics of MPC, starting with the essential and intuitive idea of receding horizon control arising in day-life problems, and tracing the history of MPC from Linear Quadratic Gaussian regulation theory. A general formulation of linear MPC is then presented including state and output constraints, closed-loop stability and robustness are briefly analyzed, reference tracking is discussed and an application to an inverted pendulum problem is presented.

The second chapter provides a theoretical analysis of infinite horizon closed-loop performance and stability of closedloop trajectories with MPC. Starting from general definitions of finite-horizon and infinite-horizon optimal control problems, bounds on the closed-loop performance are obtained by Dynamic Programming (DP) techniques. Next, a thorough analysis of stability conditions for traditional tracking MPC formulations, with or without terminal conditions is presented, followed by performance analysis of recent Economic MPC formulations, also discussed later in the seventh chapter.

The third chapter provides analysis of closed-loop behavior of MPC controlled systems, using set-valued techniques, which allow one to characterize how set of solutions depend on their constitutive parameters and how these solutions evolve in closed-loop. First, a characterization of the open-loop optimal control problem and their solution is discussed, followed by asymptotic stability, recursive feasibility and robustness analyses using Lyapunov arguments. Next, set-valued control systems, which arise due to discontinuous control actions and uncertainties, are presented and analyzed.

The fourth chapter presents the foundations of stochastic MPC algorithms in which disturbances are accounted for in terms of their statistical properties. A stochastic MPC with chance constraints is initially elaborated and discussed, and then three different routes are presented to overcome the curse of dimensionality of stochastic DP: a scenario-tree based MPC in which extremal realizations of the uncertainty over the horizon are considered to generate a tree of open-loop evolutions, a formulation based on polynomial caos expansion to propagate the uncertainty along the trajectory, and stochastic tube-based MPC approach extending a technique initially developed for robust MPC.

The fifth chapter reviews moving horizon estimation (MHE) formulations, which compute the estimate of state and disturbance trajectories using a batch of output measurements by solving a finite-horizon optimization problem in which constraints on states and disturbances are taken into account. The chapter discusses recent robust asymptotic stability results and presents a chemical reaction example in which physically unrealistic state estimates are obtained with standard techniques such as Extended and Unscented Kalman filters, whereas MHE provides consistent state estimates.

The sixth chapter further elaborates on stochastic MPC by addresses formulations of so-called dual control in which the control signal is computed not only to solve a stochastic optimal control problem but also in a way that the uncertainty in reconstructing the system state is minimized. The chapter 
proceeds with three examples of dualized stochastic control, and is concluded with an overview of possible routes to improve the numerical tractability of dual stochastic MPC formulations.

The seventh chapter presents a detailed description and analysis of economic MPC, in which the cost function is arbitrary and the closed-loop behavior may or may not be asymptotically stabilizing around an equilibrium depending on dissipativity conditions. Various formulations are presented for deterministic systems, such as with/without terminal ingredients, with average constraints in which average performance is optimized. Finally, a tube based economic MPC able to cope with uncertainties is outlined.

The eight chapter is focused on tracking approaches for MPC. The chapter starts from the standard problem of (fixed) setpoint stabilization providing theoretical guarantees, and then it discusses how trajectory tracking first and then path following can be achieved, possibly despite external disturbances. All three cases are exemplified by means of a robotic application, and the chapter ends with a sketch of a formulation that includes an economic objective term along with one of the previous cases.

A number of hybrid MPC problems and formulations are discussed in the ninth chapter. The analysis covers different problems, starting from the case of piece-wise affine state dynamics, moving to mixed (continuous and discrete) state dynamics, then addressing the case of systems with memory and logic variables, and concluding with various periodic hybrid systems. Particular attention is posed on the type of underlined optimization problem, to obtain possibly a mixedinteger linear or quadratic programming problems. The chapter ends with a general analysis of robustness issues that arise in Hybrid MPC due to the possibly discontinuous control law.

The tenth chapter discusses MPC theory and implementation for nonlinear systems described by polynomial discrete-time functions, leading to optimization problems in which objective function and constraints are polynomials. It then shows how suitable semidefinite relaxations, based on Sum-Of-Squares, can be devised for efficient online or offline (explicit) control solution. The chapter ends with a discussion on how nonpolynomial systems can be approximated by polynomial ones using Taylor's expansion.

The eleventh chapter discusses implementation strategies of MPC for large-scale systems by means of decentralized/distributed architectures. The chapter begins by describing methods for subdividing the overall system into (overlapping or non-overlapping) subsystems. Then, it describes a decentralized MPC in which interactions among subsystem are disregarded, distributed cooperative MPC in which interactions are considered to achieve global optimization and stability, or dealt with using a robust approach for non-cooperative MPC.

The twelfth chapter further elaborates on MPC large-scale scalable systems with a specific focus on the possibility of adding/removing subsystems, a problem highly motivated by recent Industry 4.0 paradigm of cyber-physical systems. The methodologies are cast within a decentralized MPC framework: stability guarantees are achieved by treating subsystem interactions as unknown disturbances and devising suitable tubebased robust MPC strategies. Conditions for plug-and-play addition/removal are analyzed, and the chapter ends with two examples of power systems and smart grids.

\section{Part II: Computations}

The first chapter of Part II reviews numerical methods for linear MPC problems, which require one to solve a convex quadratic programming $(\mathrm{QP})$ problem, at each decision given the current system state. The chapter presents the optimality conditions, then discusses how efficient interior-point strategies can be implemented by Riccati-like recursion. Next, the parametric nature of the QP is analyzed to discuss how the optimal set of active constraints evolve and how information obtained at previous decision times can be exploited within an active set QP solver. The chapter ends with a brief overview of available software.

The second chapter addresses numerical methods to solve general (nonconvex) MPC problems, which arise by the use of nonconvex cost function, constraints or nonlinear dynamics. Starting from continuous-time dynamics, after discretization, the problem is posed as a parametric NonLinear Programming (NLP) problem, whose optimality conditions are analyzed. Then, the chapter provides an overview of sequential quadratic programming and interior-point methods for NLPs, discusses the main discretization strategies, and then analyzes the parametric aspects of the NLP, warm-start and convergence, and the structure of involved matrices.

The third chapter analyzes techniques useful to convert originally nonconvex optimization problems into convex approximations that facilitate real-time application. The chapter first addresses the case of linear dynamics with nonconvex constraints, and it presents recent iterative methods based on successive convexification. Convergence analysis is presented, and then the chapter digs into implementation aspects for onboard real-time application.

The fourth chapter focuses on linear MPC problems and analyzes the associated parametric Linear Programming (LP) or parametric QP, depending on the choice of cost function. Starting from the optimality conditions, the piece-wise affine structure of the solution is revealed and so-called explicit MPC algorithms are obtained. Next, the chapter considers the case in which some integer variables occur, leading to parametric mixed-integer LP or QP problems, for which solution algorithms are discussed. The chapter ends with a discussion of computational complexity of both offline solution generation and an online computational effort.

The fifth chapter describes real-time implementation of explicit MPC by analyzing several complexity reduction approaches. The main goal is reduce the number of regions that define the piece-wise affine explicit MPC law to alleviate both memory storage and online evaluation time, with or without approximation of the optimal solution. After analyzing the linear MPC case, the chapter addresses the nonlinear MPC case in which a parametric QP problem is solved at each decision time, but its parametric components are updated to obtain stability guarantees.

The sixth chapter addresses numerical methods to solve robust MPC problems. Starting from a general case where the model dynamics are subject to unknown disturbances, a 
min-max robust MPC problem is derived, and the associated numerical challenges are identified. Then, approximated solution strategies are discussed within different specific contexts, starting from uncertain linear systems and then moving to uncertain nonlinear systems. For the latter case, the chapter reviews robust DP methods, and then more tractable scenariotree and tube-based approaches. The chapter ends with detailed analysis of numerical methods for tube-based robust MPC and set-valued computing.

The seventh chapter discusses scenario optimization methods for stochastic MPC. Starting from uncertain linear systems, a probabilistic optimization problem is posed, and sufficient conditions for probability violation are derived to pose the basis of a scenario approach, in which an appropriate number of disturbance scenarios are selected and considered within the optimization problem. Then, the chapter describes constraint removal methods that reduce computational complexity, and finally presents a numerical example to clarify the trade-off between scenario removal and performance.

The eight chapter, which ends Part II, presents a general and detailed discussion of numerical methods for nonlinear tracking and economic MPC. After defining the structure of the parametric NLP problem to be solved at each decision time and establishing its optimality conditions, the problem is relaxed by adding slack variables to state constraints to obtain a number of nice robust stability properties. Then, the chapter proceeds with discussing economic MPC approaches in which regularization terms are included to induce closedloop stability or, alternatively, an explicit stabilizing constraint is added which enforces convergence to the equilibrium point. The chapter ends with two process control case studies.

\section{Part III: Applications}

The first chapter of Part III describes different automotive applications of MPC. The chapter presents three dynamic models for powertrain, vehicle dynamics, and energy management, highlighting specific challenges and opportunities of each control problem. Then, the chapter proceeds with details on MPC problem formulation, and numerical solution either explicit (offline) or online based. Finally, it sketches the current limitations and future perspectives of nonlinear MPC applications in the automative field.

The second chapter presents a comprehensive overview of MPC applications in healthcare. Initially, the chapter reviews the key problems that arise in the field, e.g. multivariable systems, nonlinearity, constraints, articulate goals, in order to motivate the use of MPC. Then, it presents qualitatively how MPC can be posed to address problems of ambulance scheduling, joint movement, automatic anesthesia, insuline dependent diabetes, HIV, cancer, and inflammation treatments. The chapter ends by clarifying the main obstacles and challenges to overcome for a widespread use of MPC in healthcare.

The third chapter describes the application of MPC in power electronics problems, which are characterized by switched dynamics, multivariable systems, and short computation times. Two different MPCs are considered, one in which the power converter input can be modulated, through an inverter, and one in which a finite control set is available. For both problems an explicit MPC approach is used obtain the solution map offline, and then the chapter proceeds with stability and performance analysis, and finally it discusses efficient implementation of the finite control set MPC.

The fourth chapter presents two applications of receding horizon control and estimation methods for control of ground robot and of a tricopter UAV. Both robots have been 3D printed. After a brief overview of fast nonlinear MPC and MHE formulations, the chapter proceeds by describing the system dynamics and the output feedback controller implementation for each robot. The architecture includes bidirectional data exchange over the network to improve the performance through on-board parameter update computed remotely by learning techniques.

The fifth chapter deals with the application of MPC to heating, ventilation, and air conditioning (HVAC) systems, with the main goal of taking advantage of electricity pricing and ambient temperature predictions. The chapter describes the main modeling, design and implementation aspects of MPC for HVAC systems, which include hierarchical and distributed system decomposition, load forecasting, and feedback. Results of two campus applications are presented.

The sixth chapter is focused on the application of MPC algorithms to complex electric power systems. The chapter begins by describing the complex spatial and temporal interdependencies that arise in power networks, then it reviews the current hierarchical structure in use and poses the main challenges and objectives. A multi-layer MPC structure is then proposed and (temporal and spatial) lifting techniques are described to aggregate different agents and timescales, followed by a description of relevant implementation details. The chapter ends by presenting results of four examples.

The seventh chapter presents the application of MPC to two financial problems. The chapter begins by describing models of account value dynamics, which are inherently stochastic due to highly varying stock prices. Then, the problem of portfolio optimization aimed at maximizing the account value at a future time is posed as an MPC problem to take into account diversification and turnover constraints. Next, the chapter addresses dynamic option hedging problems in which the option holder is interested in trading the stocks over time so that the account matches the payoff value at expiration time. Different MPC approaches for this problems are reviewed, and the chapter ends with an overview of future directions.

\section{AUDIENCE}

This handbook is aimed at a wide audience. From one side it is an excellent reference for graduate students, researchers and practitioners in the field of control systems, numerical optimization and engineering, who have a basic knowledge of MPC and want to understand the potentials, the challenges and the benefits of its design and application. From another side, it is an up-to-date reference for MPC research experts, both in academia and in industry, interested in addressing new MPC related problems and understanding what the main research directions are and the tremendous progresses that have been made in the field over the last decades to envisage new routes to take. 


\section{SUMMARY}

In the reviewer's opinion, the Handbook of Model Predictive Control, edited by Raković and Levine, provides a thorough and comprehensive reference of theory, implementation and application of MPC. The twenty-seven chapters, suitably organized in three parts and nicely written by well-known experts in the field, meet several competing goals: clarity of communication to a diversified audience, formal rigor and selfcontainment of the material of each chapter. At the same time, the overall collection allows the reader to have a 360 degree viewpoint of Model Predictive Control theory and practice, and to transfer the MPC principle to new problems which may be considered out of scope or out of reach without such a unique and wide reference.

\section{REVIEWER INFORMATION}

Gabriele Pannocchia is an Associate Professor in Chemical Engineering at the University of Pisa, where he teaches courses on Chemical Reaction Engineering, Process Simulation and Optimization, Process Dynamics and Control, Advanced Process Control. He received the M.S. and Ph.D. degrees in Chemical Engineering from the University of Pisa (Italy) in 1998 and 2002, respectively. He held a Visiting Associate position at the University of Wisconsin - Madison (WI, USA) in 2000/2001 and in 2008, and a Post-Doctoral position at the University of Pisa from 2002 to 2006. From 2006 to 2015 he was tenured Assistant Professor at the University of Pisa, where he became Associate Professor in 2015. His research interests include: model predictive control systems, process simulation and optimization, numerical optimization, biomedical applications of automatic control algorithms, optimal planning and control for robotic systems. He is authors of more than 100 papers in international journals, book chapters and in proceedings of international conferences. Since 2008 he is Associate Editor of the Journal of Process Control and from 2013 he is Senior Editor for the same journal. From 2018 he is Associate Editor of Automatica. Dr. Pannocchia was IPC co-chair of the IFAC Symposium DYCOPS 2013, Area CoChair/Associate Editor in IFAC DYCOPS 2016, IFAC World Congress 2017, IFAC NMPC 2018, UK Control 2018. He has been keynote speaker in several international congresses (IFAC DYCOPS 2010, IFAC NMPC 2015, IFAC DYCOPS 2016, IFAC ADCHEM 2018) and Plenary Speaker at 4th IEEE Colombian Conference on Automatic Control in 2019.

\section{REFERENCES}

[1] J. Richalet, J. Rault, J. L. Testud, and J. Papon, "Model predictive heuristic control: applications to industrial processes," Automatica, vol. 14, pp. 413-1554, 1978.

[2] C. R. Cutler and B. L. Ramaker, "Dynamic matrix control - a computer algorithm," in AIChE 86th National Meeting, Houston, TX, 1979.

[3] D. Q. Mayne, J. B. Rawlings, C. V. Rao, and P. O. M. Scokaert, "Constrained model predictive control: stability and optimality," Automatica, vol. 36, pp. 789-814, 2000.

[4] D. Q. Mayne, "Model predictive control: Recent developments and future promise," Automatica, vol. 50, no. 12, pp. 2967-2986, 2014.

[5] S. J. Qin and T. A. Badgwell, "A survey of industrial model predictive control technology," Contr. Eng. Pract., vol. 11, pp. 733-764, 2003.
[6] J. B. Rawlings, D. Q. Mayne, and M. M. Diehl, Model Predictive Control: Theory, Computation, and Design, 2nd ed. Madison, WI: Nob Hill Publishing, 2017.

[7] L. Grüne and J. Pannek, Nonlinear Model Predictive Control Theory and Algorithms, 2nd ed. Springer-Verlag, 2017. 